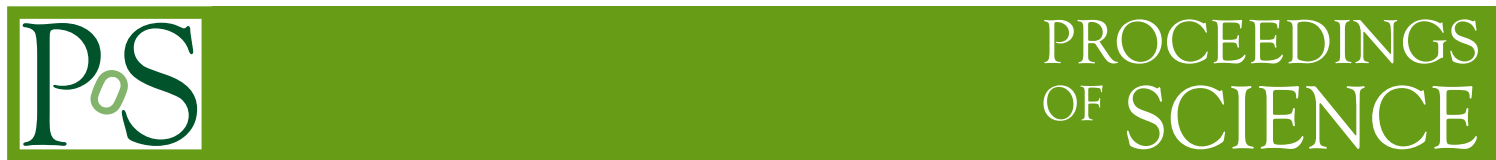

\title{
Dust in Supernovae Remnants
}

\author{
Haley Gomez* \\ School of Physics and Astronomy, Cardiff University \\ E-mail: haley.gomezeastro.cf.ac.uk
}

In this Review, I will discuss our changing view on supernovae as interstellar dust sources. In particular I will focus on infrared and submillimetre studies of the historical supernova remnants Cassiopeia A, the Crab Nebula, SN 1987A, Tycho and Kepler. In the last decade (and particularly in recent years), SCUBA, Herschel and ALMA have now demonstrated that core-collapse supernovae are prolific dust factories, with evidence of $0.1-0.7 \mathrm{M}_{\odot}$ of dust formed in the ejecta, though there is little evidence (as yet) for significant dust production in Type Ia supernova ejecta. There is no longer any question that dust (and molecule) formation is efficient after some supernova events, though it is not clear how much of this will survive over longer timescales. Current and future instruments will allow us to investigate the spatial distribution of dust within corecollapse ejecta, and whether this component contributes a significant amount to the dust content of the Universe or if supernovae ultimately provide a net loss once dust destruction by shocks is taken into account.

The Life Cycle of Dust in the Universe: Observations, Theory, and Laboratory Experiments - LCDU 2013, 18-22 November 2013

Taipei, Taiwan

\footnotetext{
${ }^{*}$ Speaker.
} 


\section{Introduction}

Supernovae $(\mathrm{SNe})$ are often seen as the bad guys in relation to the interstellar dust life-cycle, with shock waves destroying dust grains in galaxies [1], yet evidence is building that dust formation in SN ejecta may be ubiquitous. Other evidence also leads us to believe that SNe should be an important source of dust: first, without SNe there is a dust budget crisis in the Milky Way and other galaxies [2-6]. The dust produced in the cool stellar atmospheres of intermediate-mass stars, combined with current predictions for how much dust is destroyed in shocks, yields far less dust than is observed in the interstellar medium. Either another source of dust must be present to account for the observed dust in the interstellar medium (e.g. supernovae and/or interstellar grain growth), or dust destruction is negligible [4,5,7-11]. Second, without SNe as significant sources of dust, it is difficult to explain the large quantities of dust found in submillimetre (sub-mm)-selected galaxies and quasars at high redshift ( $[9,12,13]$ and references therein). There is not sufficient time for dust from evolved intermediate mass stars to form in such large quantities even when incorporating realistic and bursty star formation histories (e.g. [11,14]).

The conditions following a SN explosion are thought to be conducive to the formation of dust $[15,16]$ : the abundances of heavy elements are high, as is the density; temperatures drop rapidly in the expanding ejecta, quickly reaching levels allowing the sublimation of grain materials. Theoretical estimates (Fig. 1) predict that core-collapse SNe should produce a significant quantity of dust, approximately $0.1-2 \mathrm{M}_{\odot}$ per star (ignoring destruction, Fig. 1), depending on the metallicity, stellar mass and energy of the explosion [17-21]. These models are based on classical nucleation theory where all types of dust can form. Modelling dust formation using a chemical kinetic theory approach however, predicts lower dust masses (by roughly a factor of 10) e.g. [22-24] partly attributed to chemically modelling the type of molecules available for grain formation in the gas. Including dust destruction in the dust-formation models reduces the dust mass drastically due to sputtering in the shock waves (see the dashed line in Fig. 1 right [19]).

In this review, I will focus on what we've learnt since our previous meeting in 2008 (Cosmic Dust - Near and Far e.g. [18,25]), in particular I will describe how our understanding of the origin of dust in the nearby SN remnants (SNRs) Cassiopeia A (hereafter Cas A), Tycho, Kepler, the Crab Nebula and SN1987A has evolved over the last five years since the advent of the Herschel Space Observatory and ALMA.

\subsection{How do we detect supernova dust?}

There are a number of ways to detect or infer the presence of dust in SN ejecta, these include:

1. a decrease in the luminosity.

2. A red-blue asymmetry in optical line profiles. These originate from material moving away (on the far side of the remnant) being reddened by dust in the ejecta more than material moving towards us (self extinction).

3. The detection of onset of dust formation via an increase in IR emission;

4. Highly polarised emission from dust aligned with the magnetic field in the remnant.

Methods 1, 2 and 3 have all been applied to early-time SNRs ( $<1000$ days) e.g. [26-30]. Methods 3 and 4 provide a direct measurement of the dust mass (with the caveat of unknown grain emis- 

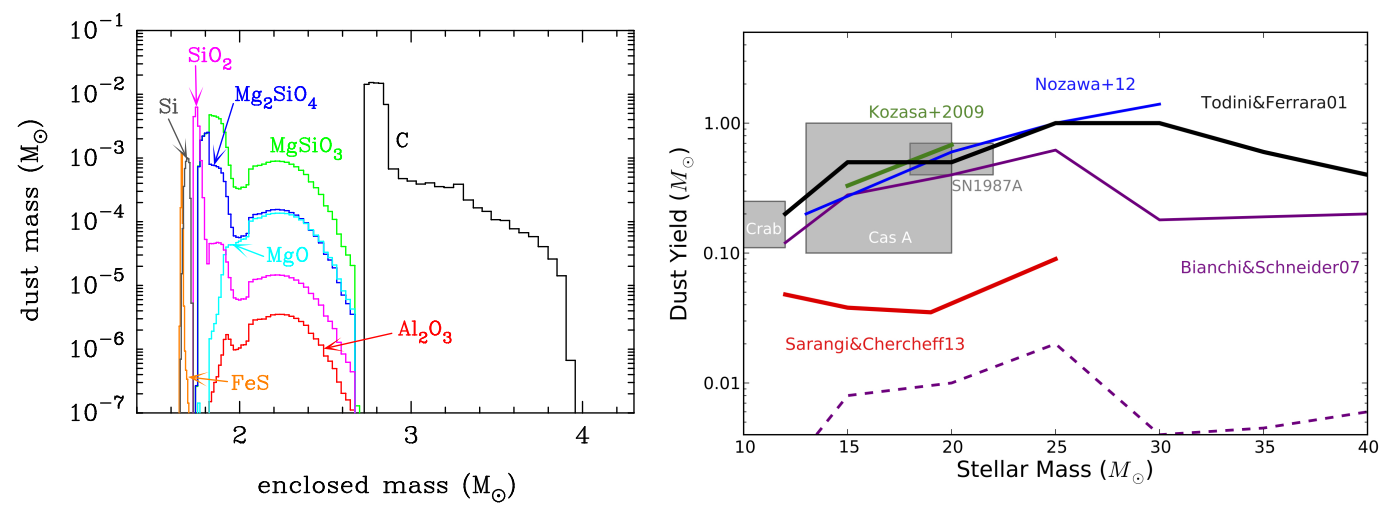

Figure 1: Left: The mass of different dust species formed in the ejecta of a theoretical SN IIb model [20]. The total mass of dust formed is $\sim 0.17 \mathrm{M}_{\odot}$. Right: A comparison of the dust yields from different theoretical supernova models in the literature verus their progenitor mass - black [17]; purple [19]; green [18]; blue [21] and red [23]. The dashed line includes dust destruction by the reverse shock [19]. The shaded regions show the observed dust masses for Cas A [32-35], the Crab Nebula [36] and SN1987A [37,38].

sivity when converting from flux to dust mass e.g. [31]). Although methods 1 and 2 only provide indirect evidence of SN dust they are particularly useful in detecting the onset of dust formation at early times. With these observational signatures, our understanding of dust in SNe and SNRs were limited to the interpretation of near and MIR studies of nearby SNRs and extremely young ( $<1500$ days) $\mathrm{SNe}$ at distances up to $20 \mathrm{Mpc}$. These observations typically found $10^{-4}-10^{-3} \mathrm{M}_{\odot}$ of warm dust (200-450 K), 1000 times lower than (i) predicted to form in theoretical models (Fig. 1) and (ii) required if $\mathrm{SNe}$ are to make a significant contribution to ISM dust in galaxies. Consequently, SNe were often dismissed as a source of dust. Of course, if cool dust at temperatures $<40 \mathrm{~K}$ exist in SNRs, it is possible that near and mid-IR measurements with telescopes such as Spitzer and AKARI would have missed this component. FIR and sub-mm observations, sensitive to emission from cooler dust grains, could therefore be extremely important in determining the total dust mass in SNRs.

\section{What we knew in 2008}

A decade ago, the Submillimetre Common User Bolometer Array (SCUBA) was used to observe the Galactic SNR Cas A. A large excess of submillimetre (submm) emission well above the extrapolation of the synchrotron component was detected (Fig. 2, 3rd panel) and interpreted as emission from $1-2 \mathrm{M}_{\odot}$ of cold $(20 \mathrm{~K})$ dust [32]. The dust was assumed to be associated with the remnant due to the high spatial correlation between the sub-mm emission and the forward and reverse shocks as traced in X-rays. Subsequently [39] used line emission and absorption towards the remnant to argue that most of the sub-mm emission in [32] arises from unrelated foreground clouds, concluding that there is no longer significant evidence for copious amounts of dust in the Cas A remnant.

Although there was clearly an issue with contamination of non-SN dust from intervening clouds along the line of sight towards Cas A, [34] reasoned that if the sub-mm emission originates within the remnant, then it would likely be polarised at a higher value than the general ISM, 

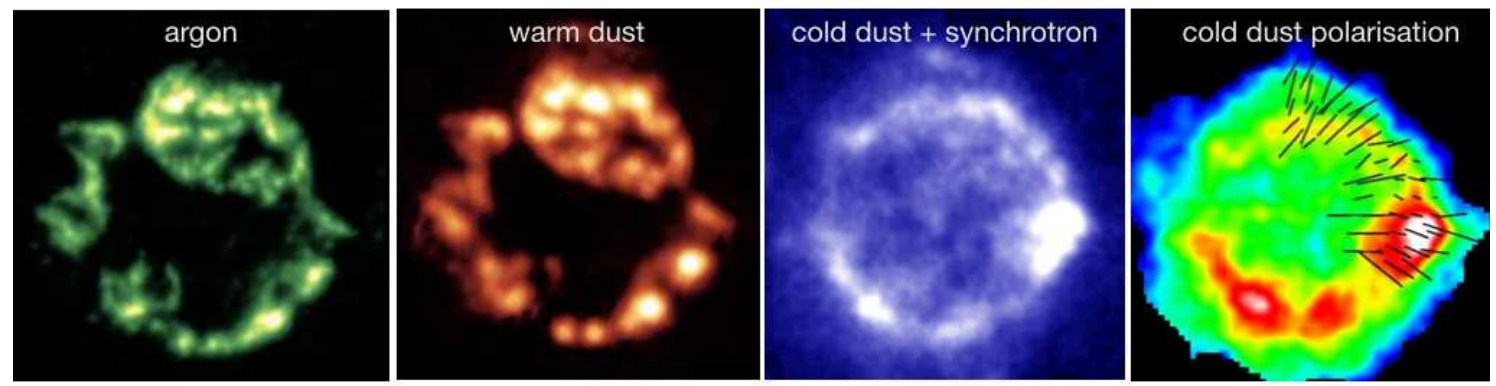

Figure 2: IR and sub-mm views of the Cassiopeia A remnant. From left to right: The Spitzer IRS observations of shocked ejecta gas (argon, green) and warm dust (orange) from [33]. The similarity between the two not only suggests that the warm dust is formed from the ejecta material, but is also being heated by the reverse shock. $850 \mu \mathrm{m}$ (blue) image with SCUBA [32] (note this emission contains both thermal dust emission and non-thermal synchrotron radiation). $850 \mu \mathrm{m}$ after subtraction of the synchrotron component with sub-mm polarisation vectors overlaid [34].

and the polarisation vectors would trace the magnetic field of Cas A. However, if the sub-mm flux originated from line-of-sight spiral arm material, then one would expect the vectors to be more-orless randomly orientated (with typical interstellar polarisation fractions of 2-7\%). Consequently, observations of Cas A were made with the SCUBA polarimeter to determine the level and direction of polarisation from the sub-mm emission - see final panel in Fig. 2. [34] found that the sub-mm emission is polarised to an unprecedented fraction of 30\%. They tested whether this could be due to contamination from synchrotron polarised signal creeping in at the longest sub-mm wavelengths, but found that the polarised signal seen in the radio is far lower, at only $3.7 \%$ on average. Assuming that only the polarised flux seen in the SCUBA map (Fig. 2) is within the remnant, then the revised dust mass in the Cas A ejecta is still a massive $\sim 1 \mathrm{M}_{\odot}$. Unfortunately, the whole of the remnant was not mapped with the polarimeter as SCUBA was taken offline shortly after these observations were taken, in preparation for its successor SCUBA-2.

With no further access to SCUBA or high sensitivity observations in the sub-mm, there was much interest in investigating the warm dust emission seen in SNRs (emitting at $24-70 \mu \mathrm{m}$ ). Detailed Spitzer observations of Cas A revealed dust emission peaking at $21 \mu \mathrm{m}$ (Fig. 2, second panel) with remarkably similar structure and location to the shocked argon ejecta [33] (Fig. 2, first panel). The mass of dust responsible for this component was estimated to be $0.02-0.054 \mathrm{M}_{\odot}$ at temperatures of $60-120 \mathrm{~K}$. These results were (at the time) the first unambiguous identification of more than $>10^{-3} \mathrm{M}_{\odot}$ of dust in the SN ejecta.

Despite evidence pointing towards larger dust masses in historical remnants compared to the near-IR estimates made previously, valid questions were asked by the community. These included questioning what appeared to be the uncomfortably large dust masses estimated from the sub-mm emission for the Cas A remnant. Whether it could be possible, for example, that the dust seen was actually formed in the massive star (pre-SN) or simply swept-up ISM? Some questioned whether a dust mass of the order of a solar mass was unphysical since it is similar (if not exceeding) the predicted mass of metals in core-collapse ejecta (Table 1). Other possible causes proposed for the high dust masses included flux contamination from line emission from both the gas in the mid-FIR and $\mathrm{CO}$ in the sub-mm. Or whether the dust emissivity in the ejecta (via differences 


\begin{tabular}{ccccccc}
\hline SNR & type & $\begin{array}{c}\text { age } \\
(\mathrm{yrs})\end{array}$ & $\begin{array}{c}\text { progenitor mass } \\
\left(\mathrm{M}_{\odot}\right)\end{array}$ & $\begin{array}{c}\text { ejected metal mass } \\
\left(\mathrm{M}_{\odot}\right)\end{array}$ & $\begin{array}{c}\text { dust mass } \\
\left(\mathrm{M}_{\odot}\right)\end{array}$ & Ref \\
\hline 1987A & $\mathrm{II}^{a}$ & 27 & $18-20^{a}$ & $1.3-3.0^{g}$ & $0.4-0.7$ & {$[37],[38],[63]$} \\
$\mathrm{Cas} A$ & $\mathrm{IIb}^{b}$ & 333 & $13-20^{b}$ & $0.7-3.0^{g}$ & $0.1-0.7$ & {$[35],[33],[34],[44]$} \\
Crab & ${\mathrm{IIn}-\mathrm{P}^{c}}$ & 960 & $8-10^{c}, 9-12^{f}$ & $0.4-0.8^{g}$ & $0.1-0.25$ & {$[36]$} \\
& & & & & & \\
Kepler & $\mathrm{Ia}^{d}$ & 410 & N/A & $1.2-1.3^{h}$ &.. & {$[54]$} \\
Tycho & $\mathrm{Ia}^{e}$ & 442 & N/A & $1.2-1.3^{h}$ &.. & {$[54]$} \\
\hline
\end{tabular}

Table 1: A summary of dust masses demonstrated to be associated with ejecta emission in SN1987A, Cas A, Crab, Tycho and Kepler SNRS. Also provided are the progenitor masses, ages and predicted metal yields. ${ }^{a}[64] ;{ }^{b}[65] ;{ }^{c}[66] ;{ }^{d}[67] ;{ }^{e}[68] ;{ }^{f}[69],{ }^{g}[10] ;{ }^{h}$ range of metals predicted for deflagration and detonation models in general $[70,71]$.

in composition or structure) is orders of magnitude higher than the typical 'astronomical silicate' and 'amorphous carbon' used to convert the flux into dust mass. One suggestion included iron needles/whiskers [40], though further work raised some issues with this proposal [41]. There is also the fact that it is difficult to envisage how such cold $(\sim 40 \mathrm{~K})$ dust could form and survive in the hot, harsh environment of the ejecta. As a consequence, many of us looked forward to the launch of the Herschel Space Observatory [42], an ESA led mission covering a wavelength range of 55-210 $\mu \mathrm{m}$ and $190-670 \mu \mathrm{m}$, allowing us to fully sample the FIR and sub-mm regime with unprecedented resolution.

\section{What we know now: The Herschel and ALMA era}

\subsection{Cassiopeia A}

Cassiopeia A was observed with Herschel as part of the guaranteed time programme MESS (Mass loss from Evolved StarS - PI Martin Groenewegen [43], see Fig. 3). After subtracting the nonthermal and warm dust components (as seen already by Spitzer [33]), a new cool dust component was revealed with $T \sim 33 \mathrm{~K}$ and mass $0.075 \pm 0.028 \mathrm{M}_{\odot}$ located across the central, western and southern parts of the remnant [35]. Combining this new cool component with the warm Spitzer dust yields an ejecta dust mass of $0.1 \mathrm{M}_{\odot}$. [35] found no evidence for cold dust in the Herschel data but this is compounded by the large amount of cirrus seen across the Herschel image (Fig. 3), arising from unrelated interstellar material emitting at temperatures of $\sim 20 \mathrm{~K}$. Note that this is not the same as saying there is no cold ejecta dust in Cas A, instead it is simply too difficult to distinguish SN dust from unrelated dust along the line of sight emitting at approximately the same temperature with photometric information alone. This is often a problem which plagues the Herschel observations of SNRs (see Fig. 5 and Section 3.4) and different techniques may be required to disentangle dust within the ejecta and dust towards or behind the source. The polarimetry observations (as described above) are one way to overcome this problem. The results from this study of Cas $\mathrm{A}\left(0.1 \mathrm{M}_{\odot}\right.$ of highly polarised dust) is supported by the high levels of self-extinction in observed [Fe II] lines in the remnant [44], thought to be the result of $0.5-1.0 \mathrm{M}_{\odot}$ of dust within the ejecta. 


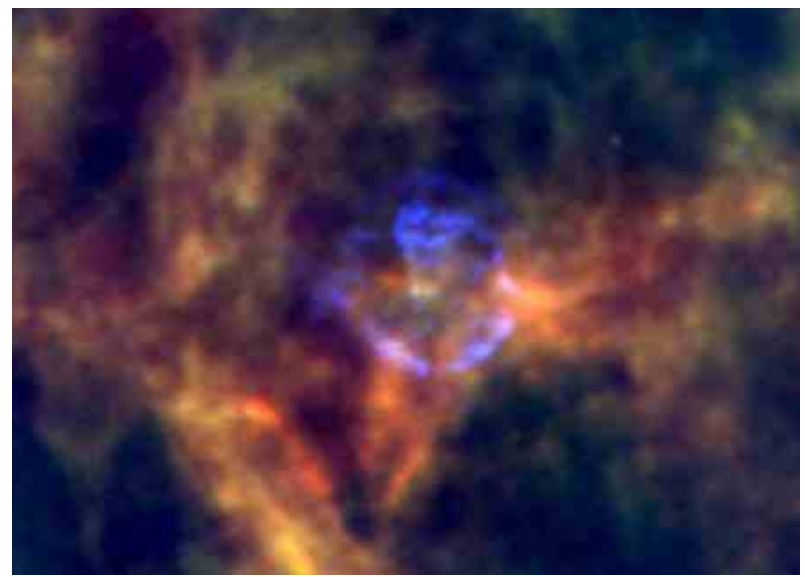

Figure 3: Three colour image of Cas A with Herschel: $70 \mu \mathrm{m}$ (blue), $100 \mu \mathrm{m}$ (green) and $250 \mu \mathrm{m}$ (red) from [35]. Note the negligible mass of hot ejecta dust shining brightly in blue (previously seen with Spitzer [33]). The faint green glow in the centre is the newly discovered cool dust component. The red emission seen all over the image is from cold dust $(\sim 20 \mathrm{~K})$, demonstrating the difficulty with disentangling emission from unrelated interstellar dust in the vicinity of, or in front of SNRs, with Herschel.

\subsection{The Crab Nebula}

Spitzer observations of the Crab Nebula found only $2.4 \times 10^{-3} \mathrm{M}_{\odot}$ of dust in the ejecta [45]. To determine if any cold dust was formed, the Crab was observed with Herschel as part of MESS (Fig. 4, [36]). Remarkably (unlike with Cas A, Tycho and Kepler - Section 3.4), the area around the remnant is relatively 'clear' from foreground or background interstellar dust. Therefore the emission seen in Fig. 4 (left) is clearly associated with dust in the Nebula. The combination of Spitzer, Herschel and Planck (observing at frequencies from 30-857GHz, [46]) allowed the midFIR-mm spectral energy distribution (SED) to be fully sampled, enabling the synchrotron emission at these wavelengths to be characterised (see Fig. 4, right). Indeed, the synchrotron power law slope was found to be steeper than previously estimated. The contamination from line emission to the mid-FIR fluxes was determined from additional Spitzer and Herschel spectroscopy (Fig. 4), contributing less than $5 \%$ to the integrated flux beyond $24 \mu \mathrm{m}$. After removing line and synchrotron emission, the remaining flux was attributed to two components of dust, a warm component with mass $10^{-3} \mathrm{M}_{\odot}$ at $63 \mathrm{~K}$ and a cool component at $34 \mathrm{~K}$ with mass $0.1-0.2 \mathrm{M}_{\odot}$ (depending on the dust composition). The dust is distributed within the well-known filaments, located in the densest ejecta gas.

Subsequent works have investigated more complex methods of deriving the dust mass in the Crab. [47] fitted the SED with a large number of modified blackbodies at a range of grain sizes (and temperatures) with a more realistic heating source. They revised the dust mass in the Crab to $0.02-0.13 \mathrm{M}_{\odot}$, with the upper end of their range consistent with the two-component fitting in [36], and the lower end suggesting an order of magnitude less dust than the previous work. However, the biggest difference in the dust masses derived in [47] is due to a choice of different optical constants compared to those used in [45] and [36] and not the multi-temperature SED modelling. Furthermore, the optical constants used to derive the $0.02 \mathrm{M}_{\odot}$ of dust (taken from [48]) have no data beyond $300 \mu \mathrm{m}$, crucial for determining the total dust mass from cold, large grains. Instead, the 

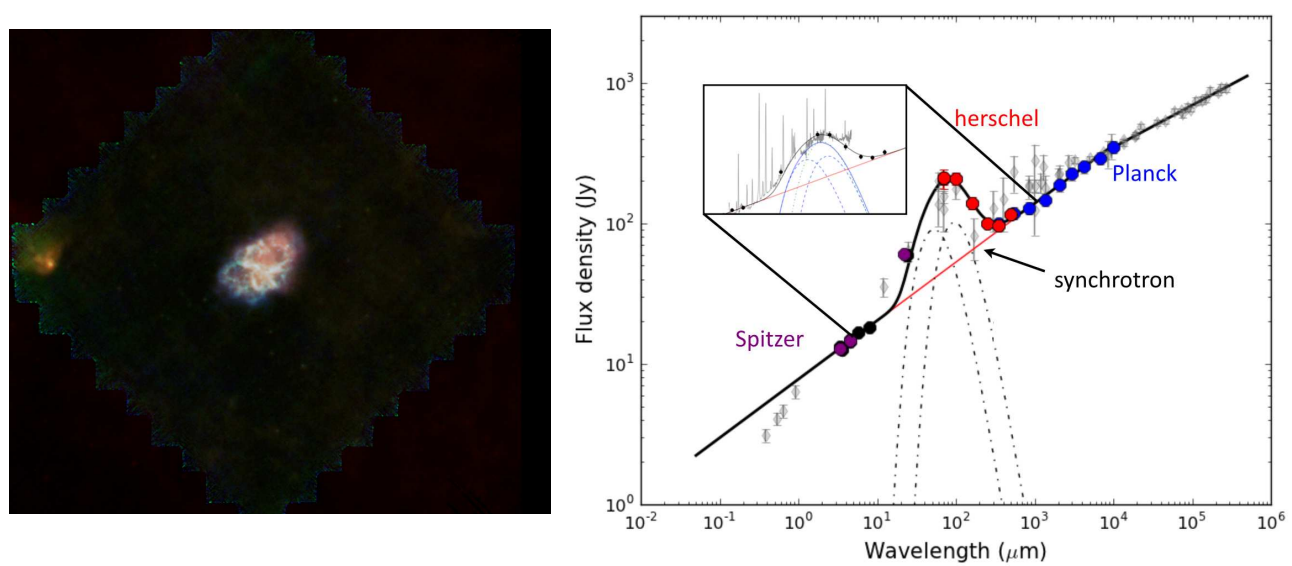

Figure 4: Left: Wide field view of the Crab Nebula with Herschel [36] demonstrating both dust emission in the remnant and the lack of cirrus in the region. The composite image consists of warm dust (blue) and cold dust (yellow and orange). These images have had the synchrotron emission subtracted. Right: The entire mid-IR - radio SED of the Crab [36] using Spitzer, Herschel and Planck photometry with literature values (grey). The inset box also includes the Spitzer and Herschel spectra and the dot-dashed lines show the warm and cool dust components.

optical constants required to model the SED in the sub-mm have been estimated by an extrapolated power-law which could severely underestimate the mass required to fit the SED. Indeed the silicate fit to the SED in [47] underestimates the observed FIR-submm fluxes (see their Section 3). Given the cut-off imposed for large grains in their model combined with the lack of optical constant data in the sub-mm, it is therefore not surprising that [47] find a lower dust mass. Recent attempts at modelling the affects of a multi-temperature SED model compared to the canonical two-component fit used in [36] shows that this creates (at most) a factor of two difference in the derived dust masses [31]. This supports the claim that using a more realistic multi-temperature grain model for the SED is not responsible for the order of magnitude decrease in dust mass suggested by [47].

Subsequent radiative transfer modelling of dust in the ejecta [49] (which encompassess varying grain size distributions, gas geometry and a more physical heating source) derives ejecta dust masses consistent with the parameters in [36] and the upper end of the range quoted in [47]. If the ejecta is clumpy, the radiative transfer models imply more dust is required to fit the SED with $0.4-0.6 \mathrm{M}_{\odot}$ of amorphous carbon grains in the debris [49]. At the time, the Herschel observations of the Crab Nebula [36] provided the cleanest view of dust in a SNR, due not only to the relatively low column density of intervening interstellar dust, but also the ability to resolve out the different emission components in order to pin down the contribution from thermal dust emission.

\subsection{SN1987A}

Spitzer observations of SN1987A ( [37] and references therein) found only $10^{-4} \mathrm{M}_{\odot}$ of warm dust originating in the ring structure (where the shockwave from the explosion is sweeping up the presupernova circumstellar material). Given the insignificant amount of dust seen previously, it was originally assumed that the remnant would not be detected at the longer Herschel wavelengths. Nethertheless, a bright unresolved source was clearly visible at the location of the remnant in the HERITAGE map of the Large Magellanic Cloud - see Fig. 5. The Herschel source was attributed to 

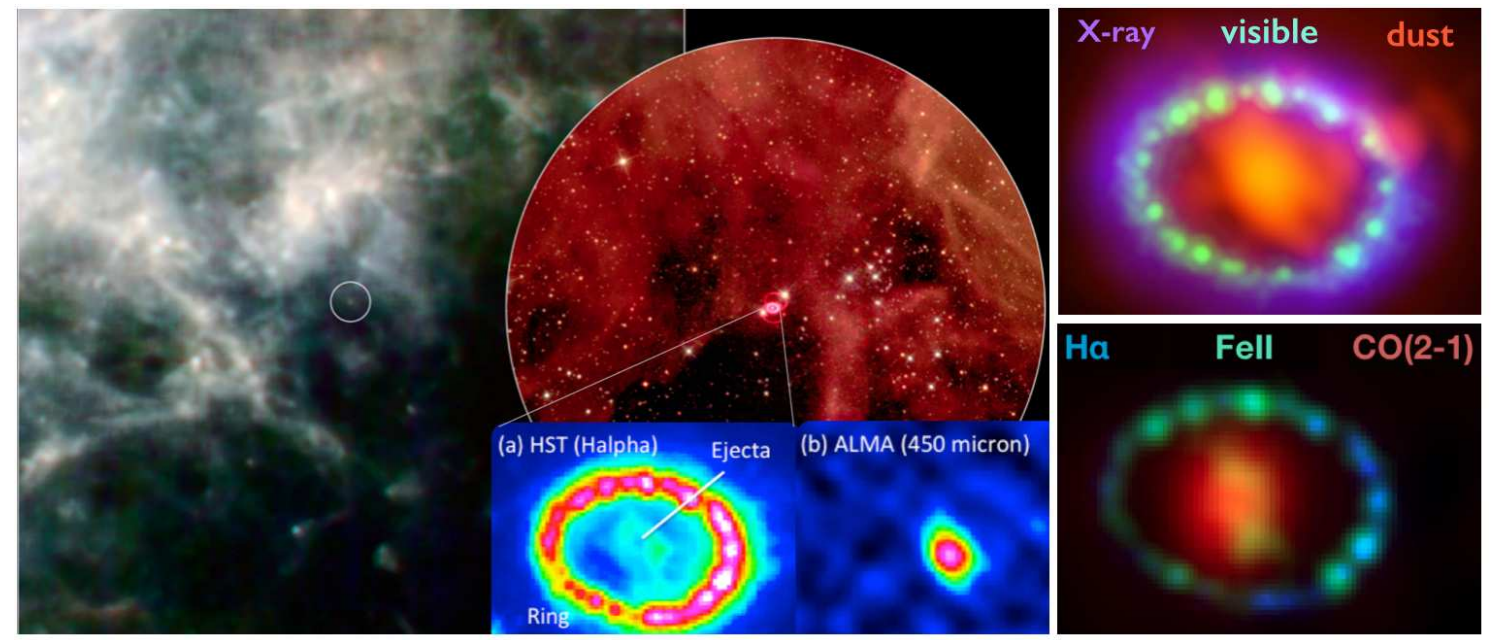

Figure 5: Left panel: Herschel composite image of $100 \mu \mathrm{m}$ (blue), $160 \mu \mathrm{m}$ (green) and $250 \mu \mathrm{m}$ (red) with the unresolved SN1987A circled. Zoomed-in HST image (ESA/NASA-JPL/Caltech/UCL) Inset (a): Further zoom in on HST view of the inner ring and the central ejecta. Inset (b): The nail in the coffin: ALMA $450 \mu \mathrm{m}$ observations of SN1987A demonstrating the cold dust emission originally detected with Herschel is resolved into the innermost ejecta region [38]. Top right: Three colour SN1987A showing the inner ejecta (red) and the outer ring. Cold dust from ALMA at $450 \mu \mathrm{m}$ (red-orange, [38]) with optical (green), and soft X-ray (blue). Bottom right: Three colour image of SN1987A with $H \alpha$ (blue), [Fe II] ejecta (green) and CO in red (ALMA, [52]). Credits: R. Indebetouw et. al., A. Angelich (NRAO/AUI/NSF); NASA/STScI/CfA/R. Kirshner; NASA/CXC/SAO/PSU/D. Burrows et al.

$0.4-0.7 \mathrm{M}_{\odot}$ of cold dust ( $\left.\sim 20 \mathrm{~K}\right)$ in SN1987A. Given such a large mass, [37] proposed this must be part of the metal-rich ejecta (just as with the Galactic remnants, the swept-up mass is predicted to be two-three orders of magnitude lower than this). This would suggest that not only is dust formation efficient in SN ejecta, but almost all of the predicted metals in the ejecta must be in the form of dust (Table 1). It became clear that higher resolution sub-mm observations would unequivocally rule out the possibility that the unresolved FIR emission seen by Herschel originates from swept-up material, line contamination, or a background source etc. The ideal opportunity to address these issues presented itself in the form of ALMA (the Atacama Large Millimeter/submillimeter Array), an interferometer observing from $400 \mu \mathrm{m}$ to $3 \mathrm{~mm}$ with resolutions of 0.7 to $4.8^{\prime \prime}$.

ALMA observations of SN1987A have since confirmed that a massive amount of dust seen initially with Herschel originates from the SN ejecta and not from another source or pre-supernova mass-loss [38]. Fig. 5 (top right, see also [50,51]) shows the three colour image of optical, X-ray and cold dust in SN1987A. The emission as seen by ALMA originates from $>0.2 \mathrm{M}_{\odot}$ of dust at $26 \mathrm{~K}$ and is clearly concentrated in the centre of the remnant: the dust has not yet been affected by the reverse shock. The ALMA observations [52] also showed that the maximum contribution from line emission to the sub-mm and mm fluxes is negligible $(<12 \%$, see also Section 4 and Fig. 5 (bottom right)). This provides unambiguous confirmation that massive amount of cold dust was formed in the ejecta within the last 20 years. 

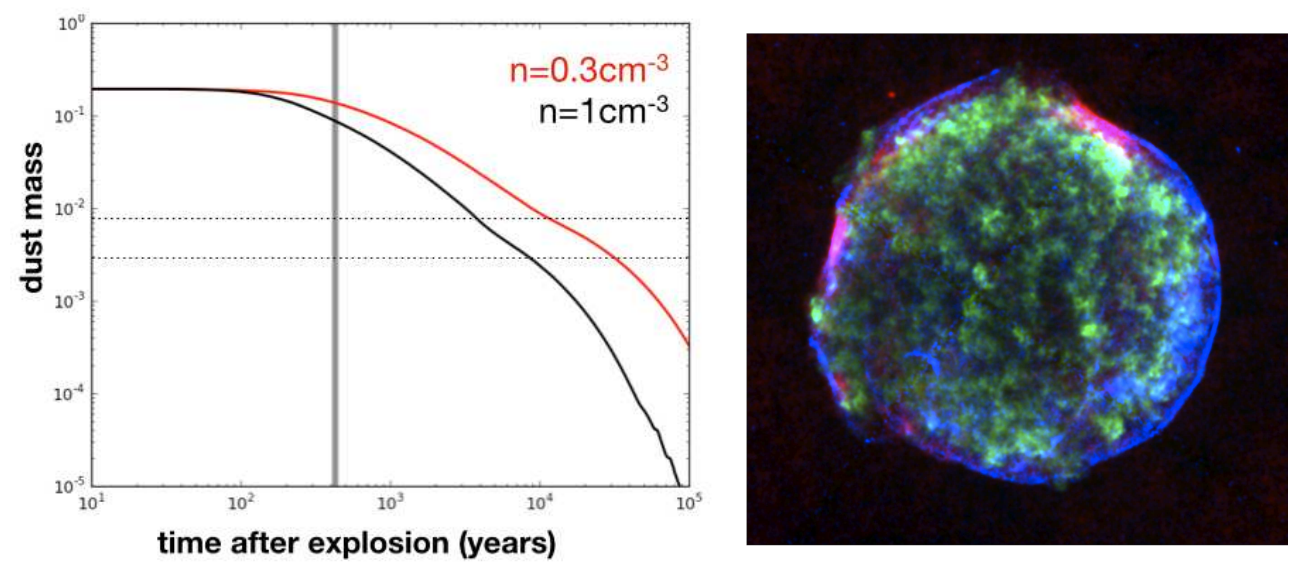

Figure 6: Left: The theoretical dust mass (in $\mathrm{M}_{\odot}$ ) formed (and destroyed) over time within a Type Ia ejecta based on the W7 deflagration model [53]. The two curves indicate ejecta expanding into interstellar gas with density $n=0.3 \mathrm{~cm}^{-3}$ (red) and $n=1 \mathrm{~cm}^{-3}$ (black). The grey vertical line represents the age range for the Tycho and Kepler SNRs and the dotted horizontal lines indicates the dust masses observed with Herschel, though these are attributed to swept up dust and not originating in the ejecta [54]. Right: Three colour image of Tycho's SNR composed of X-rays (blue - from shock-heated swept up ISM and green from the ejecta) and $8.6_{-1.8}^{+2.3} \times 10^{-3} \mathrm{M}_{\odot}$ of hot dust $(\sim 90 \mathrm{~K})$ seen by Herschel in red. Credit: NASA/CXC; ESA/Herschel/PACS/MESS Consortium/H L Gomez, created with the software package APLpy [55].

\subsection{Type Ia SNRs}

The environment of a Type Ia remnant is likely to be harsher than a core-collapse SN, with higher radioactive heating in the ejecta resulting in increased destruction of dust grains or even inhibiting the formation of dust and molecules. Furthermore, the density of the expanding shell drops more rapidly than in core-collapse ejecta, with gas densities three orders of magnitude lower which may also inhibite the condensations of dense clumps in the gas. An interesting question is whether, despite these harsher conditions, dust still forms in Type Ia ejecta. The presence of ejecta dust in these kind of explosions could affect the interpretation of SN light curves, and any lack of dust would provide crucial information on the required conditions for dust formation.

A theoretical model of dust formation in the Type Ia ejecta was presented in [53] assuming a carbon deflagration explosion with ejecta mass of $1.4 \mathrm{M}_{\odot}$ - see Fig. 6 (left). $0.2 \mathrm{M}_{\odot}$ of dust is predicted to form in this model with the grains completely destroyed over a timescale of $10^{6}$ years. Also shown in Fig. 6 is the expected evolution of dust mass for models in which the gas is expanding into ambient gas with densities $n=0.3$ and $1 \mathrm{~cm}^{-3}$, since this will affect the amount of dust destroyed in the shock waves. Using the relative ages of Kepler and Tycho (410 and 440 years) and assuming that the ejecta is expanding into an ambient gas density of $1 \mathrm{~cm}^{-3}$, the model predicts dust masses of $\sim 88$ or $84 \times 10^{-3} \mathrm{M}_{\odot}$ respectively.

As part of the MESS programme, the Galactic remnants Tycho and Kepler were observed with Herschel. Hot dust was detected 'in' both remnants (at temperatures of $80-90 \mathrm{~K}$ ) arising from dust masses of $8 \times 10^{-3}$ and $6 \times 10^{-3} \mathrm{M}_{\odot}$ respectively [54] (see also [56]). This is an order of magnitude lower than the hot dust mass predicted from the theoretical model (Fig 6). Careful comparisons with the spatial location of the hot dust in Tycho with the hot X-ray gas (Fig. 6 right) arising from 
the ejecta (green) and the shocked swept-up surrounding material (blue), indicates that the dust is only seen at the edges of the expanding bubble of gas which is ploughing through the ISM at high speeds. This tells us that the hot dust in this image was not created in the explosion itself [54] but is instead swept-up interstellar dust. This is further supported by the interaction seen between Tycho's forward shock and surrounding molecular clouds (see Fig. 10 in [54], also [57, 58]). A similar result is seen with Kepler, though in Kepler's case the mass of swept up material is so large (and given the low interstellar densities at the location of the remnant) the hot dust likely originates from swept up circumstellar dust [54].

Whether or not cold dust exists in the Tycho and Kepler SNRs [59] is more difficult to determine given the large amounts of interstellar dust detected across the maps and in front of the remnants [54] (similar to Cas A - Fig. 3). Careful comparison with the ejecta tracers in optical and $\mathrm{X}$-ray shows no evidence of sub-mm emission from cool or cold dust coincident with the debris. In summary then (and contrary to the theoretical predictions, Fig. 6 (left)), sub-mm observations of Tycho and Kepler show no evidence of dust formation (see also [60-62]). Instead, we find the observed dust emission originates from swept-up interstellar and circumstellar material respectively. It is possible that the explosions responsible for Tycho and Kepler are in fact detonation type $([57,72])$ rather than the deflagration model assumed in [53]; the predicted mass of dust formed in detonation ejecta may be very different to the model shown in Fig. 6 (left). For example, a detonation explosion has a more rapid decline in density early on and is less well mixed compared to the deflagration, with the outermost carbon-rich layer quickly burned through, severely restricting the amount of metals available for dust formation.

On a final note, Type Ia's produce most of the iron found in galaxies (ejecting $0.6 \mathrm{M}_{\odot}$ per explosion), and interstellar gas-phase depletions in the Milky Way (where iron is depleted by factors of 10-100 [73,74]) indicate that large amounts of iron is locked up in dust grains. It is therefore somewhat surprising that we find no evidence for iron dust grains in Ia ejecta. By 400 years, the reverse shocks in Tycho and Kepler have not yet swept up the innermost iron-rich layer (where one would expect iron grains could form - see Fig. 14 in [54]) so if a significant amount ( subsolar) of iron grains had condensed in these SNRs, most of them would be unshocked and cold i.e. should be clearly visible in the Herschel data (though the interstellar material seen across these images makes it difficult to rule this out). The lack of dust in Ia's compared to core-collapse remnants suggests that significantly less dust forms in this environment, placing stringent constraints on where dust and potentially cool molecules can form.

The dust masses for the historical remnants Cas A, the Crab, SN1987A, Tycho and Kepler derived from Herschel and ALMA observations are summarized in Table 1. In the last five years, we now have confirmation that significant amounts of cold dust has formed in the ejecta of Cas A, the Crab Nebula and in SN 1987A.

\section{A Serendipitous Surprise - molecular rich ejecta}

Perhaps one of the most surprising results to come out of Herschel and ALMA studies of SNRs is the presence of significant amounts of cool ejecta material in the form of cold dust and molecules, despite the harsh environment expected within the ejecta. The presence of cool molecules in SN 
ejecta would be an important diagnostic of the properties and chemistry within the ejecta and could imply inhomogeneities and clumping. Furthermore, since CO is an effective coolant, any detection of $\mathrm{CO}$ also suggests an environment which favours further molecule formation, complex chemistry and also the formation and survival of SN dust grains. (Note that as well as enhancing dust formation, the presence of molecules in the ejecta can also inhibit it e.g. [23, 24].) Recent theoretical models following the chemistry of core-collapse ejecta suggests a $15 \mathrm{M}_{\odot}$ progenitor could create $>0.1 \mathrm{M}_{\odot}$ of $\mathrm{CO}$ [22]. This places an enormous $17 \%$ of the predicted ejecta mass in cool molecular form only a few years after the explosion.

Although vibrationally excited (hot) molecules have been detected in SN ejecta since the early 90's, only recent observations have revealed the presence of cool molecules. The first overtone and fundamental bands from small amounts of hot CO was detected a few years after the SN1987A explosion [75], and more recently, in the 300-year old ejecta of Cas A [76,77]). The former result suggests the formation of $\mathrm{CO}$ is efficient even in the high-temperature ejecta at early times, and the latter suggests that $\mathrm{CO}$ survives (or continually reforms) centuries after the initial explosion. Indeed, the detection of a dense knot of $\mathrm{CO}$ in Cas A's post-shock gas by [77] clearly demonstrates that $\mathrm{CO}$ molecules dissociated by the reverse shock must have reformed in the past several years.

ALMA has since revealed rotational transitions from cold CO in SN1987A [52] (with a partial observation of $\mathrm{SiO}$ ). The molecules were seen within $1^{\prime \prime}$ of the inner debris (Fig. 5, bottom right panel), demonstrating beyond a doubt that these molecules originate from the ejecta. As the $(2-1)$ and $(1-0) \mathrm{CO}$ lines are optically thick, the observations require $>0.01 \mathrm{M}_{\odot}$ of cold $\mathrm{CO}$ (where the lower limit is derived using the optically thin assumption).

Aside from Cas A and SN 1987A, the Crab Nebula is also known to have an abundance of $\mathrm{H}_{2}$ molecules within the filaments [78], but Herschel has revealed another 'fragile' molecule in the debris. FIR- sub-mm spectra across different locations of the Crab were taken with Herschel as part of the MESS survey [43] and revealed two unknown lines at 618 and $1235 \mathrm{GHz}$ [79]. Fig. 7 shows the full spectrum with Herschel from $447-1544 \mathrm{GHz}$. The first line to be identified was the $\mathrm{OH}^{+}$ emission at $971 \mathrm{GHz}$, with radial velocities ranging from -603 and $1037 \mathrm{~km} \mathrm{~s}^{-1}$. These velocities suggest that the emission arises from different knots and filaments in the ejecta; the emission from the unidentified lines are strongest in the south of the remnant (coinciding with $\mathrm{H}_{2}$ knots and cool dust). Given the expected frequency of $\mathrm{OH}^{+}$at $971.8038 \mathrm{GHz}$ it was relatively simple to correct the two unknown lines to a 'rest' frequency of $617.554 \pm 0.209$ and $1234.786 \pm 0.643 \mathrm{GHz}$; the ratio of these lines correspond to the $(2-1)$ and $(1-0)$ transitions from a simple diatomic molecule. [79] realised (apparently on Herschel's final day of taking observations) that these lines correspond to ${ }^{36} \mathrm{ArH}^{+}$, making this the first noble gas compound discovered in space.

So the ejecta in the Crab Nebula (despite its large expansion speeds, harsh environment and hot temperatures) provides exactly the right conditions to form noble gas molecules. The proposed formation mechanism put forward in [79] is that argon produced in the supernova is ionised in the shockwaves, which also lead to the formation of cool filaments containing $\mathrm{H}_{2}$ [78] and dust [36]. The argon is then mixed in the cool gas allowing noble gas compounds to form.

\section{Conclusions}

Evidence for dust formation in core-collapse supernova ejecta has been known for some time, with 

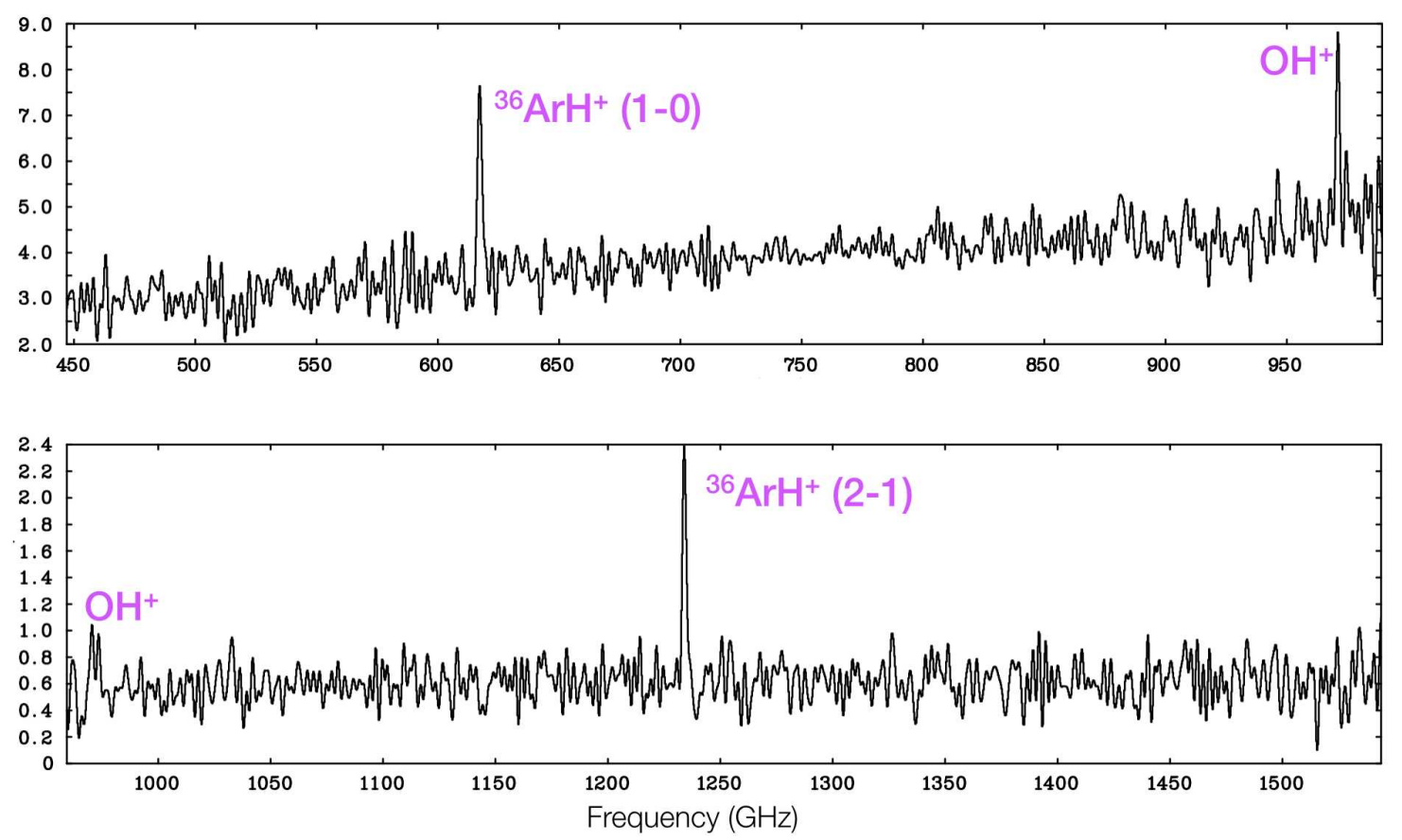

Figure 7: The Herschel FTS spectra of the Crab Nebula from [79] with top: $y$-axis flux in units of $10^{-19} \mathrm{~W} \mathrm{~m}^{-2} \mathrm{~Hz}^{-1} \mathrm{sr}^{-1}$ and bottom: $y$-axis flux in units of $10^{-18} \mathrm{~W} \mathrm{~m}^{-2} \mathrm{~Hz}^{-1} \mathrm{sr}^{-1}$. The $\mathrm{OH}$ lines indicated enabled the identification of two unknown lines at 618 and $1235 \mathrm{GHz}$ which are the $(1-0)$ and $(2-1)$ transitions of ${ }^{36} \mathrm{ArH}^{+}$respectively.

observations in the optical and near-mid IR detecting only small amounts $\left(10^{-4}-10^{-3} \mathrm{M}_{\odot}\right)$ of dust at temperatures $T>70 \mathrm{~K}$. With recent advances in sharper resolution, greater wavelength coverage and superior sensitivity in the FIR-submillimetre regime, we have learned that:

- the flux contamination in the FIR $(\lambda \geq 70 \mu \mathrm{m})$ from line emission for SN 1987A and the Crab Nebula is negligible $(<5-12 \%)$ and cannot be wholly responsible for the FIR emission seen in SNRs.

- Observations in the FIR with SCUBA, Herschel and ALMA have revealed significant amounts of cool dust is also formed in the ejecta of core collapse supernova.

- Observations and theoretical models both show that core-collapse supernova ejecta appear to provide an environment in which efficient dust and molecule formation occurs, suggestive of clumping in the ejecta.

- These works suggest that within a few hundred years after the explosion, nearly $100 \%$ of the metals predicted to be in the ejecta is in the form of dust and molecules.

- Observations of Type Ia SNRs indicate that they are not creating significant amounts of cool $\mathrm{SN}$ dust in their ejecta (caveat this is based on a sample size of two). The small mass of hot dust seen in the Tycho and Kepler remnants is not freshly-formed SN dust, instead these arise from swept-up interstellar or cirumstellar material (respectively).

The large amount of cool dust (and now molecules) therefore appears to be a significant fraction of the ejecta metals in core-collapse SNe. Of course, it is possible that some, if not all, of this freshly 
formed dust is ultimately destroyed in the shock. Some other "known unknowns" include:

- how much of a difference would incorporating 3-D models of SN ejecta make to the predicted dust mass from theoretical models?

- Does dust formation occur in the aftermath of a deflagration Type Ia explosion?

- How long is the ejecta in a molecule and dust formation phase or does this continue throughout the evolution of the remnant?

- How much dust is destroyed after encountering the reverse shock?

- What is the net yield from SN dust to the ISM?

- What can we learn from IR-submm observations of the mass, composition and chemistry of the warm and cool debris at different layers of the ejecta, in comparison with optical and X-ray-emitting ejecta material?

- What will millimetre observations tell us about the 3-D structure of the SN debris given the ability to now see the entire velocity range of the ejecta with ALMA (e.g. [52, 80, 81])?

A larger sample of FIR/sub-mm observations of SNRs, particularly with ALMA would help address some of these issues, including observing remnants with ages ranging from 1 - 300 years after the explosion. This entails building up a sample of resolved extragalactic SNRs, which requires an even greater leap in resolution and sensitivity.

\section{Next Steps}

To separate out the different components in FIR images of SNRs (which include SN ejecta, sweptup stellar winds, swept up ISM, synchrotron and line emission), we have seen that excellent sensitivity and angular resolution is required. Resolved studies also allow one to investigate the composition of the dust - for instance, in the Crab, the dust is located in regions where the ejecta is carbon-rich and is therefore likely to be composed of amorphous carbon [36]; in Cas A, the dust is located in silicate-rich ejecta and is likely proto-silicate in nature [33]. Since the emissivity of dust depends on its chemical make-up (where amorphous carbon is 2-5 times as emissive as silicate) this also has serious implications on the way we convert FIR flux into dust mass [31].

Increased sensitivity and resolution would allow us to investigate dust formation in SNRs at different stages in their evolution, by resolving young remnants $(<30 \mathrm{yrs})$ in the nearest galaxies and also intermediate-age remnants, particularly in completing the 30-300 year gap in SNR age from the current limited sample (Table 1). Current and future facilities might also offer the ability to observe and resolve dusty SNRs at even larger distances, potentially providing a statistical sample of SNRs. In order to determine what may be possible with future instrumentation, Table 2 lists the fluxes of the warm, cool and cold dust components at 20 and $100 \mu \mathrm{m}$ for Cas A, the Crab and SN1987A and predicts the fluxes and angular sizes of these SNRs out to a distance of $10 \mathrm{Mpc}$. The sensitivity and wavelength coverage of current and future missions ${ }^{1}$ are displayed in Fig. 8 with the expected mid-to-FIR SEDs of the Crab, Cas A and SN1987A as seen at a distance of $5 \mathrm{Mpc}$.

Although JWST will have 8 times the angular resolution of Spitzer MIPs with a factor of 50 improvement in sensitivity, the wavelength coverage is limited to below $30 \mu \mathrm{m}$ (Fig. 8). Given the

\footnotetext{
${ }^{1}$ http://www.stsci.edu/jwst/science/sensitivity
} 


\begin{tabular}{llccccc}
\hline SNR & Dust Model & $S_{20}$ & $S_{100}$ & $S_{20} @ 10 \mathrm{Mpc}$ & $S_{100} @ 10 \mathrm{Mpc}$ & $\theta_{10 \mathrm{Mpc}}$ \\
\hline Cas A & warm $82 \mathrm{~K}, 10^{-3} \mathrm{M}_{\odot}$ & $29 \mathrm{Jy}$ & $64 \mathrm{Jy}$ & $3.8 \mu \mathrm{Jy}$ & $7.4 \mu \mathrm{Jy}$ & $0.1^{\prime \prime}$ \\
Cas A & cool $35 \mathrm{~K}, 0.075 \mathrm{M}_{\odot}$ & $5 \mathrm{mJy}$ & $25 \mathrm{Jy}$ & $10^{-4} \mu \mathrm{Jy}$ & $2.9 \mu \mathrm{Jy}$ & $\ldots$ \\
Crab & warm $63 \mathrm{~K}, 10^{-3} \mathrm{M}_{\odot}$ & $6 \mathrm{Jy}$ & $44 \mathrm{Jy}$ & $0.2 \mu \mathrm{Jy}$ & $1.8 \mu \mathrm{Jy}$ & $0.07^{\prime \prime}$ \\
Crab & cool $34 \mathrm{~K}, 0.11 \mathrm{M}_{\odot}$ & $5 \mathrm{mJy}$ & $101 \mathrm{Jy}$ & $10^{-4} \mu \mathrm{Jy}$ & $4 \mu \mathrm{Jy}$ & $\ldots$ \\
$1987 \mathrm{~A}$ & cold $26 \mathrm{~K}, 0.4 \mathrm{M}_{\odot}$ & $0.01 \mu \mathrm{Jy}$ & $0.1 \mathrm{Jy}$ & $10^{-4} \mu \mathrm{Jy}$ & $2.5 \mu \mathrm{Jy}$ & $4 \mathrm{mas}$ \\
\hline
\end{tabular}

Table 2: The integrated fluxes at 20 and $100 \mu \mathrm{m}$ from the warm and cool dust components from the ejecta in Cas A [33,35], the Crab Nebula [36] and for the cold dust in SN1987A (estimated from the greybody fit - see Fig 3 in [38]). The predicted fluxes at 20 and $100 \mu \mathrm{m}$ from these components at a distance of $10 \mathrm{Mpc}$, along with the angular extent $(\theta)$ of the ejecta material at this distance, are also listed.

SEDs of previously published SNRs (see Table 2 and Fig. 8), JWST but will certainly be sensitive enough to detect warm/hot SN dust with temperatures $\sim 80 \mathrm{~K}$ even at distances of $5 \mathrm{Mpc}$ (Fig. 8), though the $\sim 60 \mathrm{~K} \mathrm{Crab}$ and Cas A warm dust components would not be detected above $5 \sigma$ within a 1 hour exposure beyond a distance of $100 \mathrm{kpc}$. As expected, JWST does not observe at wavelengths required to detect emission from the coldest, most massive, dust component (e.g. the $26 \mathrm{~K}$ observed in SN 1987A) even from SNRs in our own galaxy.

It is, of course, still a worthwhile endeavour to continue observing the near and MIR emission from SNRs (as demonstrated in $[33,76]$ ), but it is worth remembering that this component is only a tiny fraction of the dust mass. There is a silver lining however. The sensitivity of JWST is good enough at 11-25 $\mu \mathrm{m}$, that it could be used to detect and resolve the more massive cool dust component $\left(\sim 0.1 \mathrm{M}_{\odot}\right.$ at $\left.\sim 30-40 \mathrm{~K}\right)$ seen in the Cas A and the Crab SNRs. The expected integrated flux at $20 \mu \mathrm{m}$ from the cool Crab dust is $5 \mathrm{mJy}$ (Fig. 4, Table 2), well above the 1-hour 5- $\sigma$ sensitivity limit. However, this is only possible if there is no warm/hot dust in the same location - taking the Crab again as an example, the $20 \mu \mathrm{m}$ flux expected from the tiny mass of warm dust is $5 \mathrm{Jy}$, i.e. 1000 times brighter than the expected cool component at this wavelength. But if there are regions in the ejecta where only cool dust exists (i.e. no warm or hot dust), then JWST would be able to detect the cool dust component (though there would be no information on the peak of the dust spectral energy distribution).

To detect and characterize the very massive cold dust component, sampling of the SED beyond $100 \mu \mathrm{m}$ is essential. SPICA is a proposed Japanese-European space mission in the FIR with $3.5 \mathrm{~m}$ diameter mirror actively cooled to $4 \mathrm{~K}$. SPICA will provide a significant improvement in sensitivity compared to Herschel - by a factor of 200 in overlapping bands - but no improvement in angular resolution. SPICA will therefore be suitable for observing local (extended) sources ( $\sim$ arcminutes), but will ultimately suffer from the same resolution issue as Herschel: it will be extremely difficult to measure the dust mass in distant SNRs because of Galactic cirrus and the background emission from host galaxies. Continued (and future) observations with ALMA will address many of these uncertainties - its superior resolution and exquisite sensitivity will allow us to disentangle the different thermal and non-thermal FIR and radio components in Galactic and LMC/SMC SNRs on unprecedented scales, providing a completely different view of the ejecta (e.g. as demonstrated with Herschel for the Crab Nebula [36] and with ALMA for SN 1987A [38]). Particularly with 


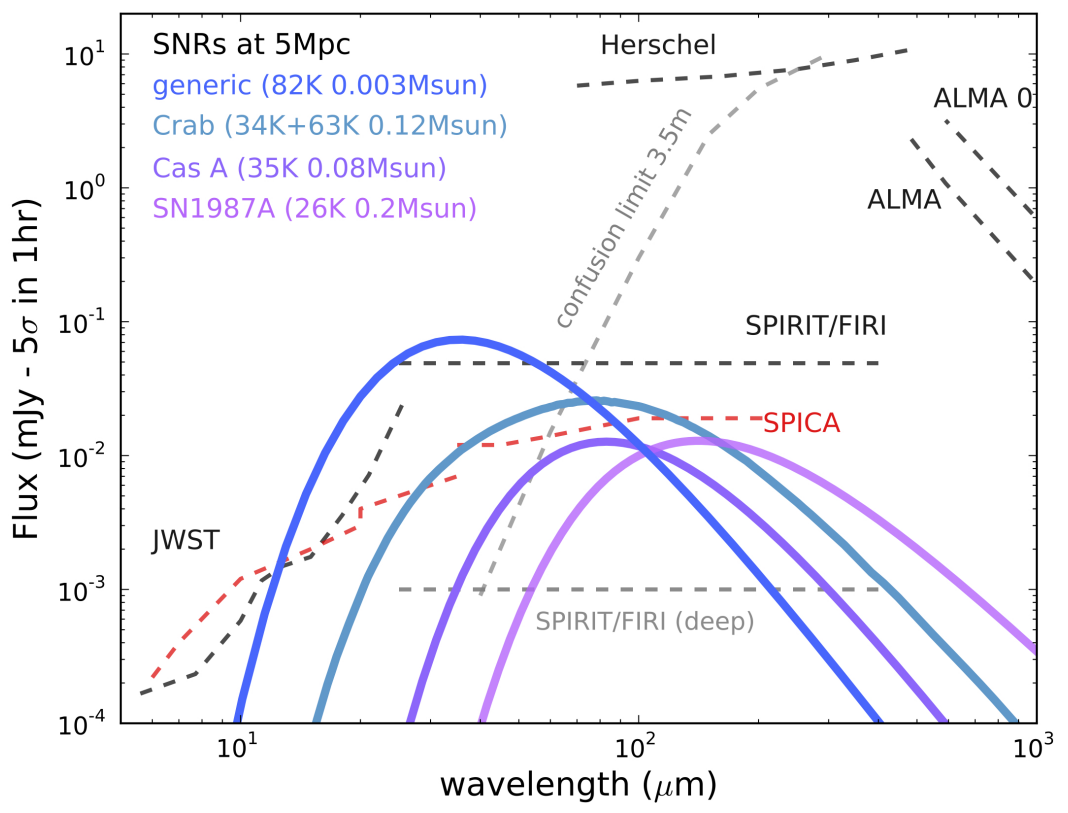

Figure 8: Sensitivity plot for current (Herschel, ALMA Cycle 0) and future MIR-FIR telescopes (JWST, SPICA, ALMA full, SPIRIT, FIRI) with 5- $\sigma, 1$ hour limit (dashed lines). The predicted SEDs from Cas A, the Crab and SN 1987A at 5 Mpc are shown. The dark blue (top) SED is predicted from a generic hot (82 K) dust model with mass $10^{-3} \mathrm{M}_{\odot}$. The light blue (second from top) SED is the total dust emission in the Crab Nebula $\left(\sim 0.116 \mathrm{M}_{\odot}\right.$ from the cool $T=34$ and warm $64 K$ components [36]). The purple SED (third from top) is the cool dust in Cas $\mathrm{A}\left(\sim 0.1 \mathrm{M}_{\odot}, T=35 \mathrm{~K}\right.$ [35]). The pink SED (bottom) is the cold dust from $\mathrm{SN} 1987 \mathrm{~A}\left(\sim 0.2 \mathrm{M}_{\odot}, T=26 \mathrm{~K}[38]\right)$.

SN1987A, ALMA can be used to further investigate the location and mass of dust, cool debris and cool molecules within the ejecta (e.g. [52]). Perhaps most excitingly, given that the SN debris is expanding at speeds of $\sim 2000 \mathrm{~km} \mathrm{~s}^{-1}$, the ejecta is beginning to collide with the ring (Fig. 5) and we can expect to see in real time the passage of ejecta (and ring) dust as these components collide. This requires continued monitoring efforts with ALMA, but potentially provides a direct test of dust destruction via SN shocks.

With respect to increasing the sample size and moving to routine extragalactic studies of SNRs in the FIR and sub-mm, improved sensitivity and sub-arcsecond angular resolution is needed between 10-500 $\mu \mathrm{m}$, particularly to overcome confusion (Fig. 8). At $10 \mathrm{Mpc}$, the Crab Nebula ejecta extends over $0.07^{\prime \prime}$ with integrated flux at of $\sim 1.4 \mu \mathrm{Jy}$ at $100 \mu \mathrm{m}$ (Table 2), requiring subarcsecond resolution and extremely high sensitivity at FIR-submm wavelengths.

ALMA will allow us to observe the cold dust in SNRs outside of the Milky Way but it will be difficult to detect SNRs at Mpc distances (Fig. 8) above the host galaxies. In its most extended configuration (when the array is completed), the resolution will range from an incredible 6-37 mas. and only in its full configuration will ALMA be able to resolve the location of the dust (whether it is ejecta or swept-up CSM and/or ISM) at such large distances. The next generation of proposed FIR space missions are therefore designed to address the need for better sensitivity, wider wavelength 
coverage and better resolution. The US-led SPIRIT [82] and EU-led FIRI [83] are proposed FIR interferometers with design characteristics of $0.02^{\prime \prime}$ resolution at $100 \mu \mathrm{m}$ and $\mu$ Jy sensitivity across the wavelength range $25-400 \mu \mathrm{m}$. Since confusion will not be a problem with these instruments, deep-field exposures $\left(\sim 10^{5} \mathrm{~s}\right)$ in the sub-mm will be possible; finally Cas A and Crab-like SNRs at $10 \mathrm{Mpc}$ will be routinely detected with FIRI at $>5 \sigma$ in typical galaxy surveys.

\section{Acknowledgments}

I'd like to thank Chris Clark, Loretta Dunne, Mikako Matsuura, Jeonghee Rho and Mike Barlow for useful discussions and Thomas Henning for provoking Section 6.

\section{References}

[1] A. P. Jones, A.G.G.M. Tielens, D.J. Hollenbach, C.F. McKee Grain destruction in shocks in the interstellar medium, ApJ 1994433797.

[2] M. Matsuura et al. The global gas and dust budget of the Large Magellanic Cloud: AGB stars and supernovae, and the impact on the ISM evolution, MNRAS 2009396981 [arXiv: 0903.1123 ].

[3] M.Boyer et al. The Dust Budget of the Small Magellanic Cloud: Are Asymptotic Giant Branch Stars the Primary Dust Source at Low Metallicity?, ApJ 201274840 [arXiv: 1201 . 5384]

[4] L. Dunne, H.L. Gomez et al. Herschel-ATLAS: rapid evolution of dust in galaxies over the last 5 billion years, MNRAS 20114171510 [arXiv: 1012.5186 ].

[5] L. Mattsson \& A.C. Andersen On the dust abundance gradients in late-type galaxies - II. Analytical models as evidence for massive interstellar dust growth in SINGS galaxies, MNRAS 201242338 [arXiv:1201.3374]

[6] L. Mattsson et al. The Herschel exploitation of local galaxy Andromeda (HELGA) VII: Strengthening the case for substantial interstellar grain growth, submitted

[7] R.S. Asano, T.T. Takeuchi, H. Hirashita. A.K. Inoue Dust formation history of galaxies: A critical role of metallicity for the dust mass growth by accreting materials in the interstellar medium, Earth, Planets, and Space 201365213 [arXiv: 1206.0817 ].

[8] E. Dwek, F. Galliano, A.P. Jones The origin and evolution of dust in high-redshift galaxies, ApJ 2007 662927 [arXiv:0705.3799].

[9] C. Gall, J. Hjorth \& A.C. Andersen Production of dust by massive stars at high redshift, A\& A Rev 20111943 [arXiv: 108.0403$].$

[10] K. Rowlands, H.L. Gomez The Dust Budget Crisis in Submillimetre Galaxies, MNRAS in press 2014 [arXiv:1403.2995]. .

[11] K. Rowlands, The dust budget crisis in high-redshift submillimetre galaxies, in proceedings of The Life Cycle of Dust in the Universe: Observations, Theory, and Laboratory Experiments, POS (LCDU2013) 011.

[12] H.L. Morgan, M.G. Edmunds Dust formation in early galaxies, MNRAS 2003343427 [arXiv:0302566].

[13] C. Gall, Sources of Dust in the Early Universe, in proceedings of The Life Cycle of Dust in the Universe: Observations, Theory, and Laboratory Experiments, POS (LCDU 2013) 008. 
[14] R. Valiante, Dust in the first quasars as a powerful probe of galaxy/BH co-evolution, in proceedings of The Life Cycle of Dust in the Universe: Observations, Theory, and Laboratory Experiments, POS (LCDU2013) 012.

[15] D. H. Wooden, D.M. Rank, J.D. Bregman, F.C. Witteborn, A.G.G.M. Tielens, M. Cohen, P.A. Pinto, T.S. Axelrod Airborne spectrophotometry of SN 1987A from 1.7 to 12.6 microns - Time history of the dust continuum and line emission, ApJS 199388477.

[16] L.B. Lucy, I.J. Danziger, C. Gouiffes, P. Bouchet Dust Condensation in the Ejecta of SN 1987A, LNP 1989350164.

[17] P. Todini \& A. Ferrara Dust formation in primordial Type II supernovae, MNRAS 2001325726 [arXiv:0009176].

[18] T Kozasa, T. Nozawa, N. Tominaga, H. Umeda, K. Maeda, K. Nomoto Dust in Supernovae: Formation and Evolution in proceedings of Cosmic Dust - Near and Far, ASP Conference Series, edited by Thomas Henning, Eberhard Grün, and Jürgen Steinacker. San Francisco: Astromomical Society of the Pacific 2009414 p.43 [arXiv: 0903.0217 ].

[19] S. Bianchi, \& R. Schneider Dust formation and survival in supernova ejecta, MNRAS 2007378973 [arXiv:0704.0586].

[20] T. Nozawa, T. Ozasa, N. Tominaga, K. Maeda, H. Umeda, K. Nomoto, O. Krause Formation and Evolution of Dust in Type IIb Supernovae with Application to the Cassiopeia A Supernova Remnant, ApJ 2010713356 [arXiv:0909.4145].

[21] T. Nozawa, et al. Supernovae as sources of dust in the early universe, Memorie della Societa Astronomica Italiana $2012 \mathbf{8 3} 77$.

[22] I. Cherchneff \& A. Sarangi Molecules in Supernova Ejecta, in proceedings of International Astronomical Union IAU Symposium 2011 280 228-236 [arXiv: 1107 . 5668].

[23] A. Sarangi \& I. Chercheff The Chemically Controlled Synthesis of Dust in Type II-P Supernovae, ApJ 2013776107 [arXiv: 1309.5887$].$

[24] A. Sarangi, Dust and molecules in the ejecta of type II-P supernovae, in proceedings of The Life Cycle of Dust in the Universe: Observations, Theory, and Laboratory Experiments, PoS (LCDU2013) 091.

[25] J. Rho et al. Dust Formation Observed in Young Supernova Remnants with Spitzer in proceedings of Cosmic Dust - Near and Far ASP Conference Series, edited by Thomas Henning, Eberhard Grün, and Jürgen Steinacker. San Francisco: Astromomical Society of the Pacific 200941422 [arXiv:0901.1699]

[26] R. Kotak et al. Dust and The Type II-Plateau Supernova 2004et, ApJ 2009704306 [arXiv:0904.3737].

[27] J.A. Andrews et al. SN 2007od: A Type IIP Supernova with Circumstellar Interaction, ApJ 2010715 $541[\operatorname{arXiv}: 1004.1209)]$.

[28] J. Fabbri et al. The effects of dust on the optical and infrared evolution of SN 2004et, MNRAS 2011 418, 1285 [arXiv:1110.3166].

[29] W.P.S. Meikle et al. A Spitzer Space Telescope Study of SN 2003gd: Still No Direct Evidence that Core-Collapse Supernovae are Major Dust Factories, ApJ 2007665608 [arXiv : 0705 . 14 39].

[30] T. Szalai, J. Vinkó, Z. Balog, A. Gáspár, M. Block, L.L Kiss Dust formation in the ejecta of the type II-P supernova 2004dj, A \& A 201152761 [arXiv: 1012 . 2035]. 
[31] L. Mattsson, H.L. Gomez, A. Andersen, M. Matsuura, From flux to dust mass I: Does the grain-temperature distribution matter for estimates of cold dust masses in supernova remnants?, in preparation

[32] L. Dunne, S.A. Eales, R. Ivison, H. Morgan, M.G. Edmunds Type II supernovae as a significant source of interstellar dust, Nature 2003424285 [arXiv: 0307320].

[33] J. Rho, T. Kozasa, W.T. Reach, J.D. Smith, L. Rudnick,T. DeLaney, J.A. Ennis, H. Gomez, A. Tappe, Freshly Formed Dust in the Cassiopeia A Supernova Remnant as Revealed by the Spitzer Space Telescope, ApJ 2008693 L39 [arXiv: 0709.2880$].$

[34] L. Dunne et al. Cassiopeia A: dust factory revealed via submillimetre polarimetry, MNRAS 2009394 1307 [arXiv:0809.0887].

[35] M.J. Barlow et al. A Herschel PACS and SPIRE study of the dust content of the Cassiopeia A supernova remnant, $A$ \& A 2010518 L138 [arXiv: 1005 . 2688].

[36] H.L. Gomez et al. A Cool Dust Factory in the Crab Nebula: A Herschel Study of the Filaments, ApJ 2012a 76096 [arXiv:1209.5677].

[37] M. Matsuura et al. Herschel Detects a Massive Dust Reservoir in Supernova 1987A, Science 2011333 1258 [arXiv:1107.1477].

[38] R. Indebetouw et al. Dust Production and Particle Acceleration in Supernova 1987A Revealed with ALMA ApJ 2014782 L2 [arXiv: 1312 . 4086$].$

[39] O. Krause, S.M. Birkmann, G.H. Rieke, D. Lemke, U. Klaas, D.C. Hines, K.D. Gordon No cold dust within the supernova remnant Cassiopeia A, Nature 2004432596 [arXiv : 0412092 ].

[40] E. Dwek The Detection of Cold Dust in Cassiopeia A: Evidence for the Formation of Metallic Needles in the Ejecta, ApJ $2004 \mathbf{6 0 7} 848$ [astro-ph/ 0401074 ].

[41] H.L. Gomez (née Morgan), L. Dunne, S.A Eales, E.L. Gomez, M. G. Edmunds, Iron Needles in Supernova Remnants?, MNRAS 20053611012 [astro-ph/ 0505557 ].

[42] G. Pilbratt et al. Herschel Space Observatory. An ESA facility for far-infrared and submillimetre astronomy, $A \&$ \& 2010518 L1 [arXiv: 1005.5331$].$

[43] M.A.T. Groenewegen et al. MESS (Mass-loss of Evolved StarS), a Herschel key program, A \& A 2011 $526162[\operatorname{arXiv}: 1012.2701]$.

[44] B.C Koo, Dust in Cassiopeia A, in proceedings of The Life Cycle of Dust in the Universe: Observations, Theory, and Laboratory Experiments, POS (LCDU2013) 087.

[45] T. Temim, G. Sonneborn, E. Dwek, R.G. Arendt, R.D. Gehrz, P. Slane, T.L. Roellig Properties and Spatial Distribution of Dust Emission in the Crab Nebula, ApJ 201275372 [arXiv: 1205 . 2062]

[46] J.A. Tauber et al. Planck pre-launch status: The Planck mission, A \& A 2010520 A1

[47] T. Temim \& E. Dwek The Importance of Physical Models for Deriving Dust Masses and Grain Size Distributions in Supernova Ejecta. I. Radiatively Heated Dust in the Crab Nebula, ApJ 20137748 [arXiv:1302.5452].

[48] F. Rouleau \& P.G. Martin Shape and clustering effects on the optical properties of amorphous carbon, ApJ 1991377526

[49] P. Owen, The Mass of Dust in the Crab Nebula: RT Models with Smooth and Clumped Dust Distributions, in proceedings of The Life Cycle of Dust in the Universe: Observations, Theory, and Laboratory Experiments, POS (LCDU2013) 020. 
[50] M. Meixner, Herschel and ALMA measurements of Dust and Molecules in Supernova 1987A, in proceedings of The Life Cycle of Dust in the Universe: Observations, Theory, and Laboratory Experiments, POS (LCDU2013) 090.

[51] R. Wesson, Dust in the remnant of SN1987A - when, where and how it formed, in proceedings of The Life Cycle of Dust in the Universe: Observations, Theory, and Laboratory Experiments, POS (LCDU2013) 021.

[52] J Kamenetzky et al. Carbon Monoxide in the Cold Debris of Supernova 1987A, ApJ 2013773 L34 [arXiv:1307.6561].

[53] T. Nozawa, K. Maeda, T. Kozasa, M. Tanaka, K. Nomoto, H. Umeda, Formation of Dust in the Ejecta of Type Ia Supernovae, ApJ 201173645 [arXiv: 1105.0973$].$

[54] H.L. Gomez et al. Dust in historical Galactic Type Ia supernova remnants with Herschel, MNRAS 2012b 4203557 [arXiv:1111.6627].

[55] T. Robitaille \& E. Bressart APLpy: Astronomical Plotting Library in Python, Astrophysics Source Code Library 2012 [ascl : 1208 . 017]

[56] D. Ishihara et al. Origin of the dust emission from Tycho's SNR, A \& A 2010521 L61 [arXiv:1009.6047]

[57] C. Badenes, K.J. Borkowski, J.P. Hughes, U. Hwang, E. Bravo onstraints on the Physics of Type Ia Supernovae from the X-Ray Spectrum of the Tycho Supernova Remnant, ApJ 20066451373 [arXiv:0511140]

[58] A. Chiotellis, D. Kosenko, K.M. Schure, J. Vink, J.S. Kaastra Modelling the interaction of thermonuclear supernova remnants with circumstellar structures: the case of Tycho's supernova remnant, MNRAS 20134351659 [arXiv: 1307 . 8123]

[59] H.L. Morgan, L. Dunne, S.A. Eales, R.J. Ivison, M.G. Edmunds Cold Dust in Kepler's Supernova Remnant, ApJ 2003597 L33 [arXiv: 0309233].

[60] K.J. Borkowski, et al. Dust Destruction in Type Ia Supernova Remnants in the Large Magellanic Cloud, ApJ 2006642 L141 [arXiv: 0602313]

[61] B.J. Williams et al. Dust in a Type Ia Supernova Progenitor: Spitzer Spectroscopy of Kepler's Supernova Remnant, ApJ 20127553 [arXiv: 1206.1054 ]

[62] J. Johansson, R. Amanullah, A. Goobar Herschel limits on far-infrared emission from circumstellar dust around three nearby Type Ia supernovae, MNRAS 2013 431 L43 [arXiv: 1209 . 1090 ]

[63] M. Lakićević, J. Th van Loon, F. Patat, L. Staveley-Smith, G. Zanardo The remnant of SN 1987A revealed at (sub-)mm wavelengths, A \& A 2011532 L8 [arXiv: 1107.1323 ].

[64] W. D. Arnett, J.N. Bahcall, R.P. Kirshner, S. E. Woosley Supernova 1987A, Annu. Rev. Astron. Astrophys. 198927269.

[65] O. Krause, S.M. Birkmann, T. Usuda, T. Hattori, M. Goto, G.H. Rieke, K.A. Misselt The Cassiopeia A Supernova Was of Type IIb, Science 20083201195 [arXiv: 0805 . 4557].

[66] N. Smith The Crab nebula and the class of Type IIn-P supernovae caused by sub-energetic electron-capture explosions, MNRAS 2013434102 [arXiv: 1304 . 0689].

[67] S. P. Reynolds et al. A Deep Chandra Observation of Kepler's Supernova Remnant: A Type Ia Event with Circumstellar Interaction, ApJ 2007668 L135 [arXiv : 0708 . 3858]. 
[68] O. Krause, M. Tanaka, T. Usuda, T. Hattori, M. Goto, S. Birkmann, K. Nomoto Tycho Brahe's 1572 supernova as a standard typeIa as revealed by its light-echo spectrum, Nature $2008 \mathbf{4 5 6} 617$ [arXiv:0810.5106].

[69] G.M. MacAlpine \& T.J. Satterfield The Crab Nebula's Composition and Precursor Star Mass, AJ 20081362152 [arXiv: 0806.1342 ].

[70] K. Nomoto, F.-K. Thielemann, K. Yokoi Accreting white dwarf models of Type I supernovae. III Carbon deflagration supernovae, ApJ 1984286644

[71] C. Badenes, E. Bravo, K.J. Borkowski, I. Domínguez Thermal X-Ray Emission from Shocked Ejecta in Type Ia Supernova Remnants: Prospects for Explosion Mechanism Identification, ApJ 2003593 358 [arXiv:astro-ph/0304552]

[72] S. Park et al. A Super-solar Metallicity for the Progenitor of Kepler's Supernova, ApJ 2013767 L10 [arXiv: 1302.5435]

[73] E. Jenkins, A Unified Representation of Gas-Phase Element Depletions in the Interstellar Medium, ApJ 20097001299 [arXiv:0905.3173]

[74] E. Jenkins, Dust Processing in the Interstellar Medium, in proceedings of The Life Cycle of Dust in the Universe: Observations, Theory, and Laboratory Experiments, POS (LCDU2013) 005.

[75] J. Spyromilio, W.P.S. Meikle, R.C.M. Learner, D.A., Allen Carbon monoxide in supernova 1987A, Nature 1990334327

[76] J. Rho, T.H. Jarrett, W.T. Reach, H. Gomez, M. Andersen, Carbon Monoxide in the Cassiopeia a Supernova Remnant, ApJ 2009693 L39 [arXiv : 0901 . 2308].

[77] S.H.J. Wallström et al. CO rotational line emission from a dense knot in Cassiopeia A. Evidence for active post-reverse-shock chemistry, A \& A 2013558 L2 [arXiv: 1309.4229$].$

[78] E.D. Loh, J.A. Baldwin, Z.K. Curtis, G.J. Ferland, C.R. O’Dell, A.C. Fabian, P. Salomé A Survey of Molecular Hydrogen in the Crab Nebula, ApJS 201119430 [arXiv: 1103.6043 ]

[79] M.J. Barlow et al. Detection of a Noble Gas Molecular Ion, ${ }^{36} \mathrm{ArH}^{+}$, in the Crab Nebula, Science 20133421343 [arXiv: 1312 . 4843].

[80] T. DeLaney et al. The Three-dimensional Structure of Cassiopeia A, ApJ 20107252038 [arXiv:1011.3858]

[81] K. Isensee et al. The Three-dimensional Structure of Interior Ejecta in Cassiopeia A at High Spectral Resolution, ApJ 20107252059 [arXiv: 1006.0918 ]

[82] D. Leisawitz et al. The Space Infrared Interferometric Telescope (SPIRIT): High- resolution imaging and spectroscopy in the far-infrared, J. Adv. Space Res. 200740689 [arXiv: 0707.0883 ].

[83] F. Helmich and R. Ivison FIRI - a Far-Infrared Interferometer, Experimental Astronomy 200723245 [arXiv:0707.1822]. 